\section{Protection of the airway with the LMA during upper airway surgery}

To the Editor:

We read with interest the recent publication by Ahmed and Vohra ${ }^{1}$ and we agree with their conclusions that the laryngeal mask airway (LMA) provides a safe and reliable airway, although our findings of LMA contamination were rather different.

Following a personal communication with one of the authors (Vohra) we incorporated their scoring system into a study that we were conducting involving the use of laryngeal masks in outpatient pediatric dental surgery.

We recruited 71 patients (ASA I or II, age range 2-15 yr) who were undergoing routine extractions for carious teeth under general anesthesia. The patients were anesthetized with propofol $\left(3-4 \mathrm{mg} \cdot \mathrm{kg}^{-1}\right)$, or via an inhalational induction $\left(\mathrm{O}_{2} / \mathrm{N}_{2} \mathrm{O} /\right.$ sevoflurane $)$ depending on the ease of venous access and patient preference/compliance. Anesthesia was maintained by spontaneous ventilation with $\mathrm{O}_{2} / \mathrm{N}_{2} \mathrm{O} /$ sevoflurane. Following the extraction of teeth by experienced dental surgeons the LMA was removed when the patients were fully awake. The LMA was immediately examined by one of the authors (Dolling) and scored accordingly. ${ }^{1}$

Our results are markedly different (Table). Shortlived desaturations below $94 \%$ were seen in ten of our patients during recovery [three with score 1 , four with score 2, three with score 3 (no statistical significance)]. All patients uneventfully met our discharge criteria.

Several $\operatorname{articles}^{2,3}$ have shown that the LMA protects the larynx from fluids above but this may not always be the case. We used standard, rather than reinforced LMAs, which may be associated with increased movement of the LMA during surgery and subsequent reduction in the integrity of the protective seal. Also, our surgeons used only dental packs to stem the flow of blood. This is likely to result in more blood in

TABLE Comparison of LMA soiling scores in our study with Ahmed/Vohra's

\begin{tabular}{lll}
\hline Soiling score $^{*}$ & Dolling/Anders/Rolfe & Ahmed/Vohra \\
\hline 0 & $1.4 \%(1)$ & $87 \%$ \\
1 & $52.1 \%(37)$ & $11 \%$ \\
2 & $38.1 \%(27)$ & $2 \%$ \\
3 & $8.4 \%(6)$ & $0 \%$ \\
\hline
\end{tabular}

LMA $=$ laryngeal mask airway. ${ }^{*} 0=$ no blood; $1=$ staining on the cuff; 2 = staining on inside of mask; 3 = blood in the tube. the airway than in nasal or septal surgery, with subsequently increased pooling above the cuff. An alternative explanation, of the differing results, could simply be that the LMAs were contaminated by intra-oral blood on removal (unlike the Ahmed/Vohra study, no intra-oral suction was performed prior to removal to minimize stimulation).

It would be interesting to clarify the reasons for our different results using reinforced LMAs or a fibreoptic scope to examine the underside of the LMA prior to its removal.

Stuart Dolling MB BS FRCA

Nicola R.K. Anders MB CHB FRCA

Sian E. Rolfe MB CHB FRCA

Stockport, UK

\section{References}

1 Abmed MZ, Vohra A. The reinforced laryngeal mask airway (RLMA) protects the airway in patients undergoing nasal surgery - an observational study of 200 patients. Can J Anesth 2002; 49: 863-6.

2 John RE, Hill S, Hughes TJ. Airway protection by the laryngeal mask. A barrier to dye placed in the pharynx. Anaesthesia 1991; 46: 366-7.

3 Samarkandi AH, Ali MS, Elgammal M, Bakbamees HS. Airway protection by the laryngeal mask airway in children. Middle East J Anaesthesiol 1995; 13: 107-13.

\section{REPLY:}

We thank Dolling et al. for their comments and appreciate this opportunity to reply. We note fundamental differences in the methodology of the study by Dolling and ours: ${ }^{1}$

- rigid vs reinforced laryngeal mask airway (LMA);

- oral vs nasal surgery;

- spontaneous vs controlled ventilation;

- pediatric vs adult patients;

- no suctioning vs suctioning at the end of the procedure.

All of which may have contributed to the differing results.

The LMA protects the airway by covering it and movement of the LMA will reduce this protection. Oral surgery and a rigid LMA are likely to result in increased LMA movement. Increased LMA movement, combined with the negative pressure created by spontaneous breathing, will encourage blood to breach the LMA barrier and contaminate it from the inside.

The shape of the LMA is based on adult anatomy and pediatric masks are the downsized version of adult masks. It can be postulated that pediatric masks do not make an 
effective seal because of the differences between adult and pediatric upper airway anatomy. We are not aware of any work on this. Finally, if secretions or blood are not cleared at the end of the procedure, this is likely to result in increased LMA contamination upon withdrawal.

We have also observed that overinflating the LMA cuff distorts its shape and reduces the area of contact with the pharynx, thus worsening the seal. We do not know how the authors assessed the adequacy of the seal in their study. This may be an additional factor explaining higher contamination scores.

We agree that further studies using reinforced LMA and direct scoring with endoscopic examinations may clarify the situation.

\section{Zubair Ahmed MB BS FRCA}

Akbar Vohra MB CHB DA FRCA

London, UK

\section{Reference}

1 Abmed MZ, Vobra A. The reinforced laryngeal mask airway (RLMA) protects the airway in patients undergoing nasal surgery - an observational study of 200 patients. Can J Anesth 2002; 49: 863-6.

\section{Function of the aperture bars on the $L M A$}

To the Editor:

I read with great interest the letter of Drs. Al-Shaikh and Pilcher indicating that the epiglottic retention aperture bars on the Classic laryngeal mask airway (LMA; Laryngeal Mask Company Ltd., Oxon, U.K.) were without apparent useful function. ${ }^{1}$ A new disposable Portex LMA (Portex Ltd., Kent, U.K.), which does not have epiglottic retention aperture bars, has been recently introduced. I have inserted and then fibreoptically examined the position of the new disposable Portex LMA in ten patients. In all patients, the new Portex LMA worked acceptably well as a ventilatory device. However, I found that in most of these patients the epiglottis entered the breathing shaft of the aperture bar-less Portex LMA, which made it difficult to identify supralaryngeal and laryngeal anatomy. In order to identify anatomy and intubate the trachea fibreoptically in these patients, I had to pull the Portex LMA back approximately 0.5 to 1.0 $\mathrm{cm}$; the LMA pull back allowed disengagement of the epiglottis from the breathing shaft of the LMA (of varying degrees from partial to total) and identification of the anatomy. Although the new Portex epiglottic retention aperture bar-less LMA has the advantage of allowing one full size greater endotracheal tube to pass through the breathing shaft (compared to the same sized Classic LMA), the lack of aperture bars is a distinct disadvantage. It is hoped that this communication will stimulate others to further examine the function of aperture bars.

Jonathan L. Benumof MD

San Diego, California

\section{Reference}

1 Al-Shaikh B, Pilcher D. Is there a need for the epiglottic bars in the laryngeal mask airway? (Letter). 2003; 50: $203-4$.

\section{REPLY:}

Thank you for giving us the opportunity to reply to Professor Benumof who showed interest in our study. ${ }^{1}$ We have shown that the absence of the epiglottic bars in the laryngeal mask airway (LMA)-Classic ${ }^{\mathrm{TM}}$ has no effect on either its clinical performance (in 380 patients) or the fibreoptic endoscopic view of the larynx (in 80 patients). The new disposable Portex Soft Seal ${ }^{\mathrm{TM}}$ LM has been in use for some time in Europe. It is deliberately designed without epiglottic bars and with an internal "bowl" that has a larger internal volume to ensure that any epiglottis that may fall into it would not obstruct the lumen of the tube. A study comparing the performance of a size 4 Portex Soft Seal ${ }^{\mathrm{TM}}$ LM with a size 4 LMA-Classic ${ }^{\mathrm{TM}}$ in 200 spontaneously breathing adult patients undergoing various surgical procedures has shown no difference in the clinical performance and the endoscopic view of the larynx in both groups. ${ }^{2}$ The incidence of sore throat in the early postoperative period was significantly lower in the Portex Soft Seal ${ }^{\mathrm{TM}} L M$ group. This can be either (or both) due to the absence of the epiglottic bars or the limitation to the rise in intra-cuff pressure during $\mathrm{N}_{2} \mathrm{O}$ anesthesia in the Portex Soft Seal ${ }^{\mathrm{TM}} L M$ group due to the very low permeability of the Soft Seat ${ }^{\mathrm{TM}}$ cuff material to $\mathrm{N}_{2} \mathrm{O}$.

Baha Al-Shaikh FFARCSI

Ashford, Kent

\section{References}

1 Al-Shaikh B, Pilcher D. Is there a need for the epiglottic bars in the laryngeal mask airway? (Letter). 2003; 50: 203-4.

2 Van Zundert AAJ, Fonck K, Al-Shaikh B, Mortier E. A comparison of the LMA-Classic ${ }^{\mathrm{TM}}$ with the new disposable Soft Seal ${ }^{\mathrm{TM}}$ LM in spontaneously breathing adult patients. Anesthesiology 2003; (in press). 